\title{
Predicting corrosion related signatures
}

\author{
R. Adey \& J. M. W. Baynham \\ C M BEASY Ltd, Ashurst Lodge, Southampton, UK
}

\begin{abstract}
Computer simulation has been widely used to predict the corrosion related electric and electromagnetic signatures of naval vessels. The modelling strategy has varied from simple dipole type models to detailed boundary element models of the vessel and its environment. For the dipole models users have had to choose the location and strength of the dipoles based upon experience, using range data or data from similar vessels. Whereas the boundary element model enables the user to define the actual geometry of the vessel, the electrochemical properties of the materials and the properties of the environment as data to the model and obtain predictions of the electric and magnetic signatures.

This paper describes the simulation tools developed as part of the BEASY software to predict electric and magnetic signatures. Comparison is made between results of the boundary element detailed models and the dipole type models.
\end{abstract}

\section{Introduction}

The design of a cathodic protection system is of interest to defence organisations not only to ensure the integrity of the ship but also because of the electric fields generated in the sea water by the Cathodic Protection (CP) system. These fields are known as the signature of the ship.

Electromagnetic signatures are playing an important role in the detection of naval vessels and in the fusing of intelligent mines. The static electric signature is the electric field associated with the DC corrosion or cathodic protection current that flows through the seawater around a vessel. This is sometimes referred to as the Underwater Electrical Potential or UEP. The corrosion related magnetic (CRM) field is the coupled magnetic field caused by the corrosion related electric currents flowing in the seawater between the anodes and the ship hull. 
It is important to note that UEP and CRM signatures exist even in the absence of a cathodic protection system. They are caused by the galvanic potential differences between the metallic structures in contact with the seawater. For example, the relative position in the electrochemical table of steel and bronze provides a sufficient driving potential to create an electric field.

In order to control the signatures and to preserve the integrity of a vessel it is essential to be able to predict the impact of the design and operation of the $\mathrm{CP}$ system on the electric fields.

Computational models have been widely used to predict the electromagnetic fields associated with vessels due to on board systems and ferromagnetic aspects. The software BEASY [1] has been widely used to predict the performance of cathodic protection systems by modelling the coupled electric fields and electrochemistry for complete ships and other structures. Other authors have used simple dipole models to make predictions. In this paper an integrated approach is presented to enable the performance of the CP system, the corrosion related electric field and the corrosion related magnetic field to be predicted.

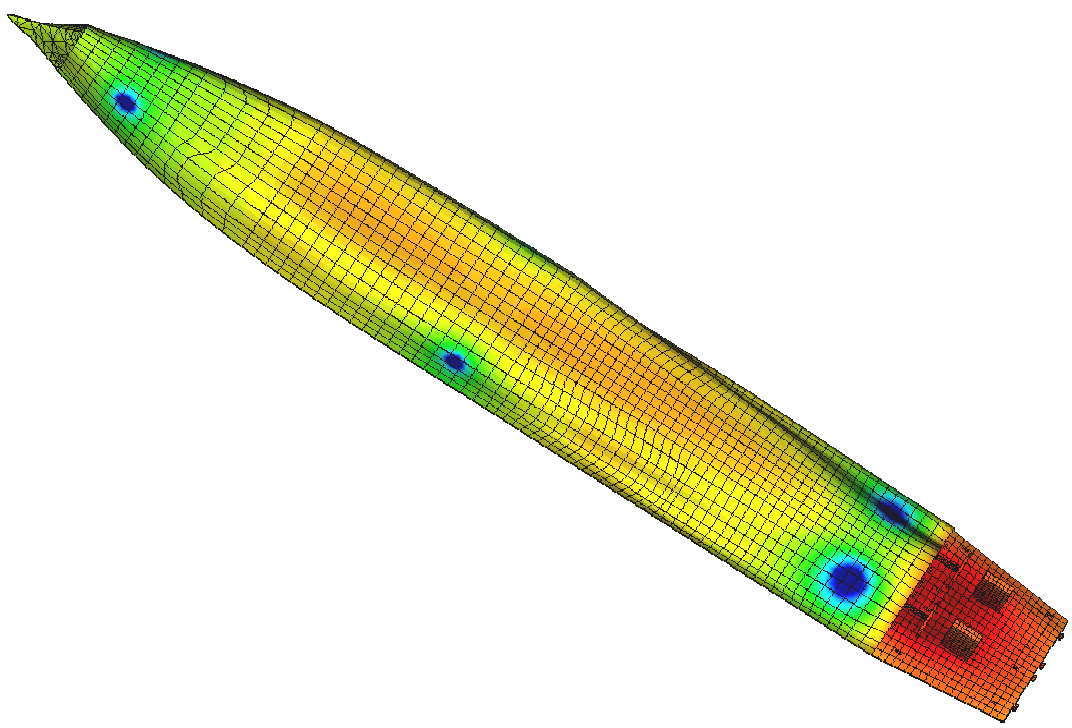

Figure 1: BEM model of a ship (note: only elements on the wetted surface of the ship).

\subsection{Theoretical aspects}

The ability of the boundary element method (BEM) to provide excellent solutions to problems associated with corrosion simulation has been discussed in [2]. The BEM requires the user to describe only the boundary or surface of the ship to be modelled, thus simplifying the modelling process. Other benefits are described in [2]. Figure 1 shows an example of a BEM model of a ship hull, including propeller shafts, rudders, and propellors. 
To represent a CP problem the computer model must simulate the IR drop through the electrolyte and the electrochemical electrode kinetics on the metallic surfaces. BEM solution of the Laplacian in the sea water, combined with representation of the electrode kinetics associated with different surface types, has been shown to provide accurate results for the complex current fields in the sea water [3 to 7] and hence the UEP signature.

The CRM signature generated by the "volume currents" flowing in the seawater may be found by solving the vector potential equation:

$$
\nabla^{2} \underline{A}=-\mu \underline{J}
$$

where

$$
\begin{aligned}
& \underline{A}=\text { vector potential } \\
& \underline{J}=\text { Vector of current density components }
\end{aligned}
$$

but this approach is computationally expensive.

Recently an alternative approach has been used, in which the CRM is obtained by means of an integration over the boundaries of the volume through which the volume currents are flowing. This approach, introduced by Allan [8], is significantly less expensive, and is used in the examples shown in this paper.

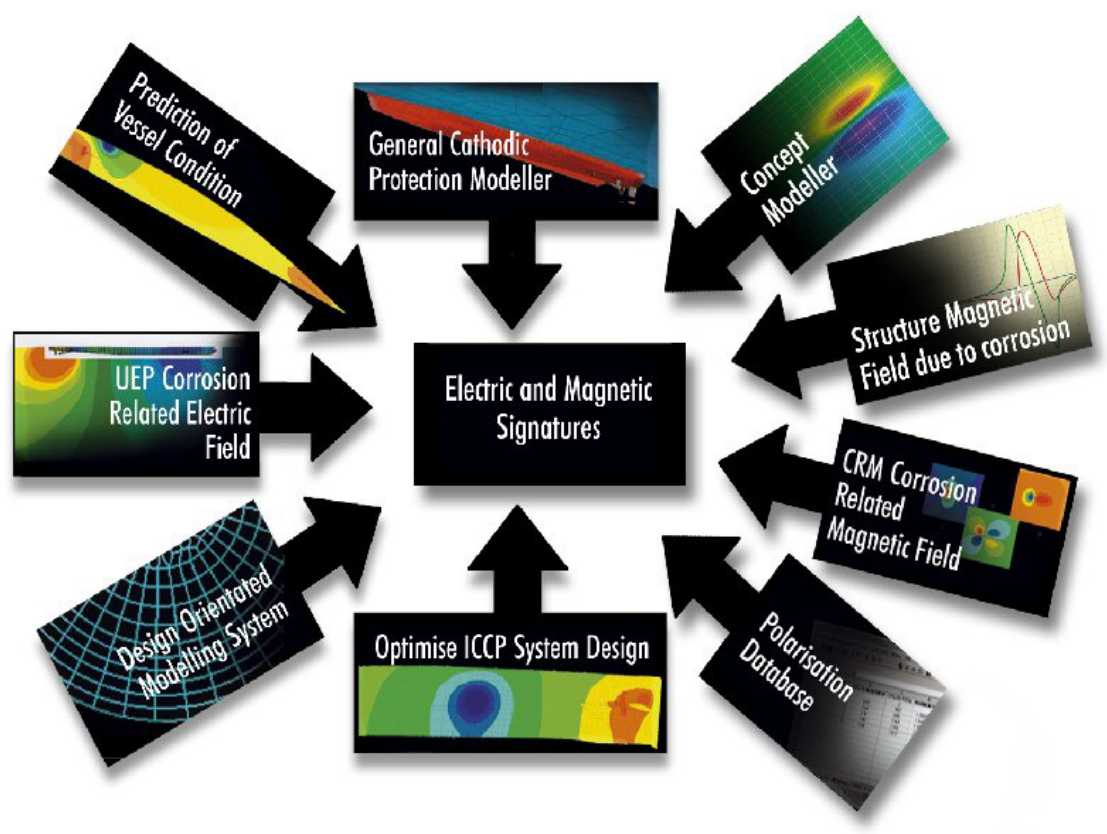

Figure 2: View of the simulation tools available to compute the components which make up a vessels signature. 


\subsection{Signatures}

The corrosion related signature of a vessel is made up of two components, the electric field and the magnetic field. The electric field is calculated directly as part of the BEM solution of the corrosion model. The magnetic field due to the currents flowing through the sea water can be computed once the electric field is known.

However this is only part of the magnetic signature as there is also current flowing through the metal structure of the vessel back to the power supply for an ICCP system or back to the anode. The magnetic field due to this current flow through the vessel must be added to the magnetic field caused by the volume currents to obtain the total electromagnetic field.

\section{Signature simulation tools}

The signature tools available within BEASY are as follows.

- Detailed BEM modeller capable of predicting the corrosion related electric and magnetic fields generated by the currents flowing through the sea water and sea bed.

- Structure magnetic field modeller capable of predicting the magnetic field generated by the currents flowing through the ships structure.

- Dipole modeller capable of predicting the electric and electromagnetic fields generated by a vessel approximated by a set of dipoles

- Characterisation tool capable of generating a set of dipoles to match an electric and magnetic field obtained from ranging or the detailed BEM model.

\subsection{Detailed BEM modeller}

The BEM model computes the electric field surrounding the vessel as part of the solution of the Laplace equation and the metal electrode kinetics equations. The magnetic field is computed using the known current densities to perform a boundary integration.

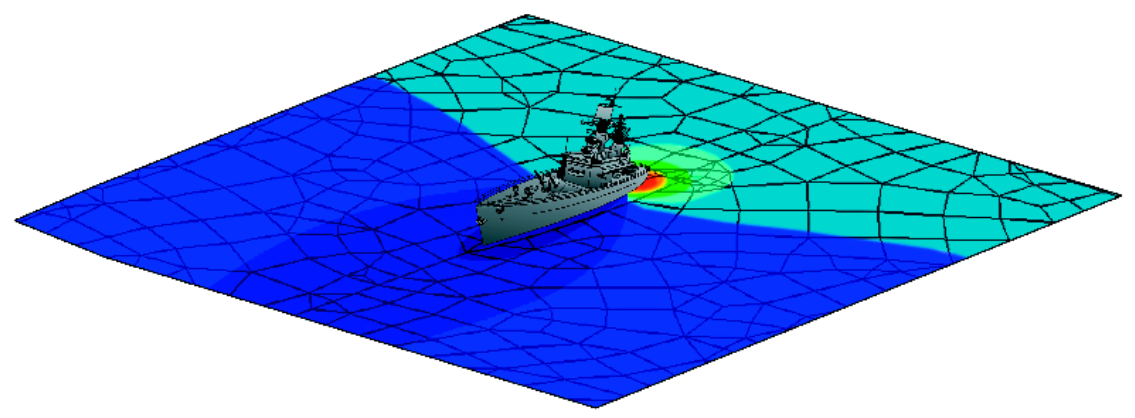

Figure 3: The BEM modeller can predict the electric field on the BEM mesh, and the electric and magnetic fields at internal point positions. 


\subsection{Structure magnetic field model}

The internal structure of the vessel is idealised as a series of wires and the current flowing in each wire is determined from the current densities (on the surface of the vessel) which have been obtained using the detailed BEM model.

The user simply selects the structure and the software automatically calculates the current flowing. The magnetic fields generated by each wire are determined using Biot-Savart.

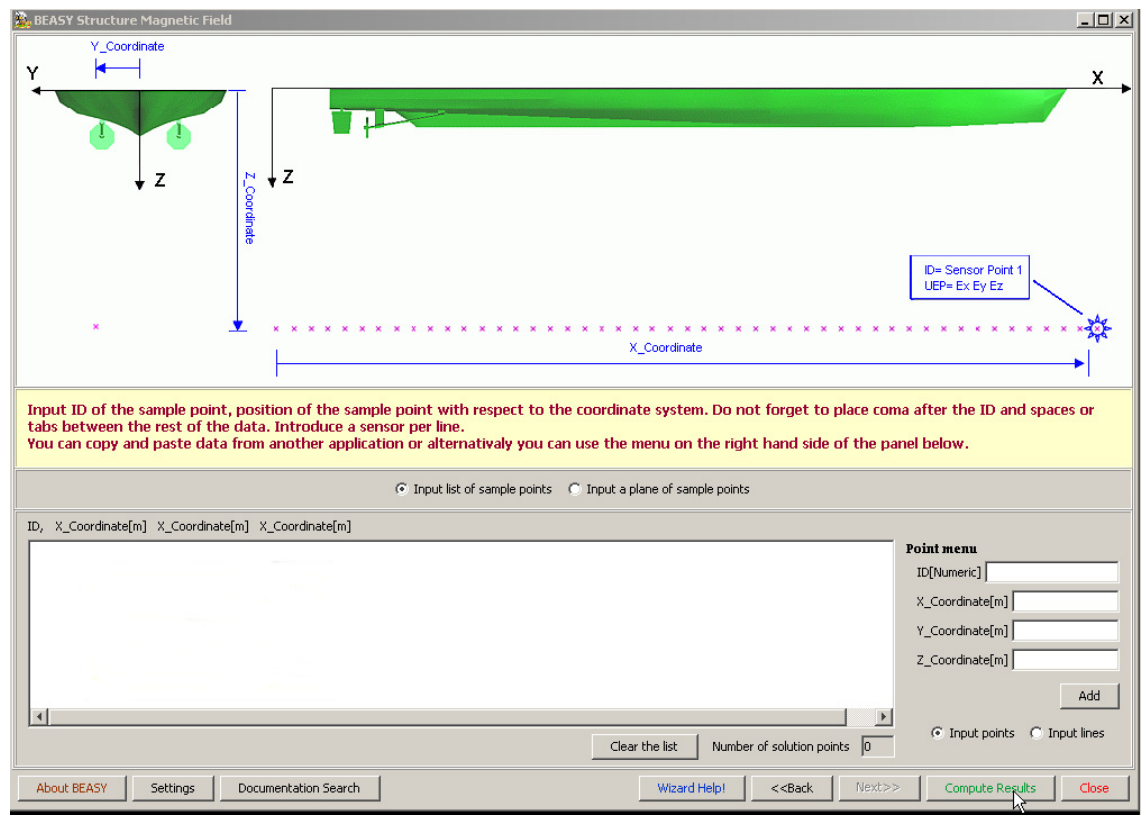

Figure 4: View of the Structure Magnetic Field software.

\subsection{Dipole simulation tool}

In contrast to the modelling tools described above (which use the detailed current distributions caused by the real hull shape), the dipole simulation tool requires the user to specify the current flowing in each dipole, and the position of each dipole source and sink.

The dipole model allows multiple horizontal dipoles, and uses a two-layer solution (in which the depth of layer 1 can be selected by the user) to calculate the resulting electric and magnetic fields.

\subsection{Characterisation tool}

The characterisation tool provides a way of automatically obtaining a dipole distribution which reproduces a known electric signature. The signature may 
have come from the detailed BEM model, or could also come from experimental measurements.

The user defines the overall dimensions of the ship, and can define the depth of the top conducting layer. The software uses an optimisation technique to create the set of dipoles.

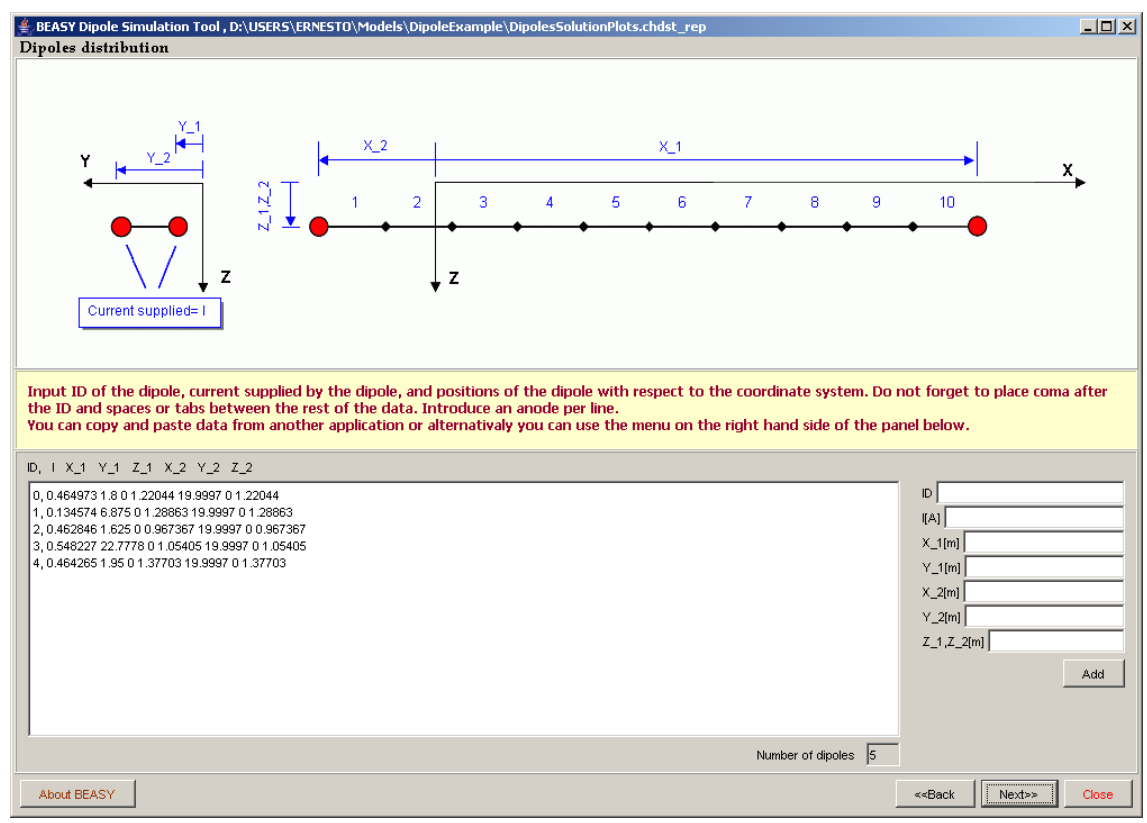

Figure 5: The user defines the position and strength of the sources and sinks used to describe the current flowing from and to the metal surfaces.

\section{Comparison}

The objective of this example is to use the various tools described above to determine the magnetic field generated by current flowing into and out of the ends of a semi-cylindrical "hull" floating at the surface of an electrolyte with conductivity of $4 \mathrm{~S} / \mathrm{m}$. The hull (shown in Figure 7) is at the surface of a volume of electrolyte with depth 1000 metres, and extending 1000 metres horizontally.

Firstly a detailed BEM model of the hull was used to determine the volume currents flowing in the electrolyte, and the currents flowing through the surface of the hull. The currents were used to determine the magnetic field caused by the volume currents, and the magnetic field caused by the currents flowing along the return path through the hull.

Next a dipole solution was used to determine the electric and magnetic fields, for a dipole with the same current, and with the source and sink separated by the same distance as the ends of the hull, i.e. at $+/-50$ metres, and at approximately the "centroid" of the anode and cathode areas. 


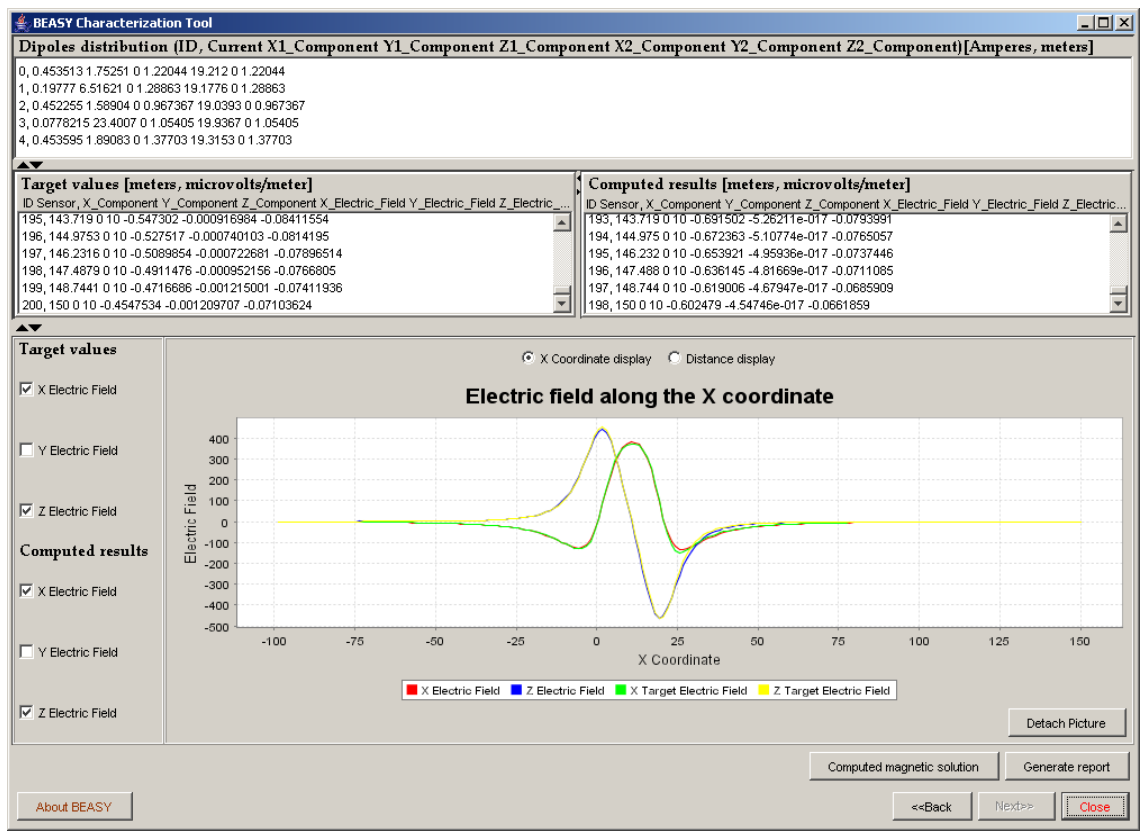

Figure 6: Comparison between electric field generated by the equivalent dipoles and the actual signature.

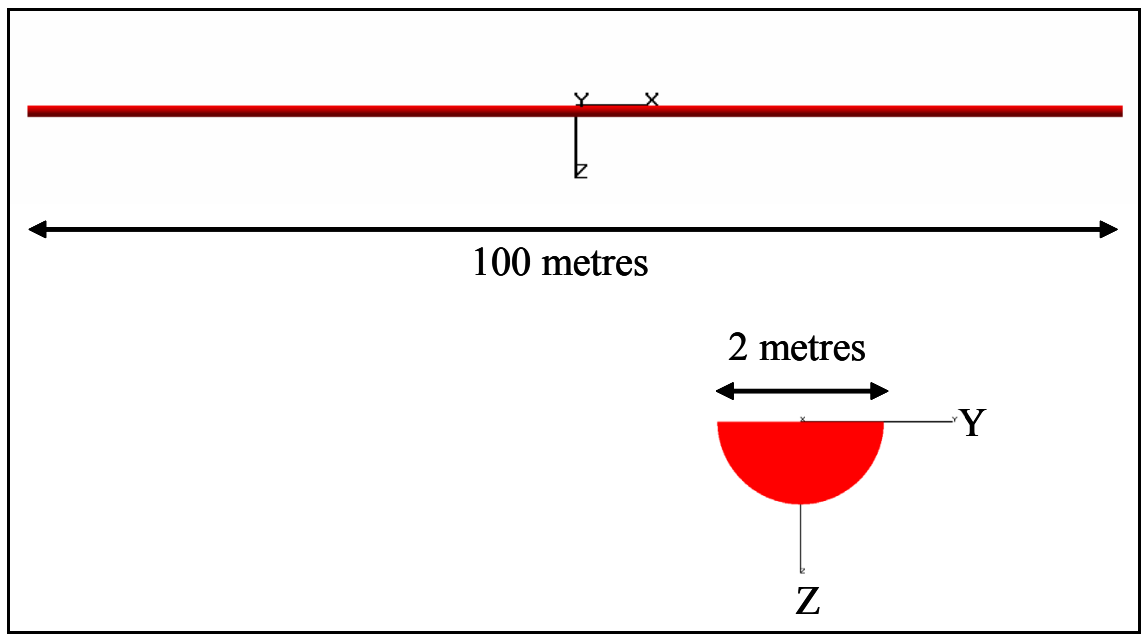

Figure 7: The "hull" used in the comparison.

One end of the hull was given an anodic current of 20 Amperes, and at the other end a polarization curve for steel (Figure 8) was used.

Results were obtained along a line of 401 internal points placed under the hull from position $(-200,0,20)$ to position $(200,0,20)$. 


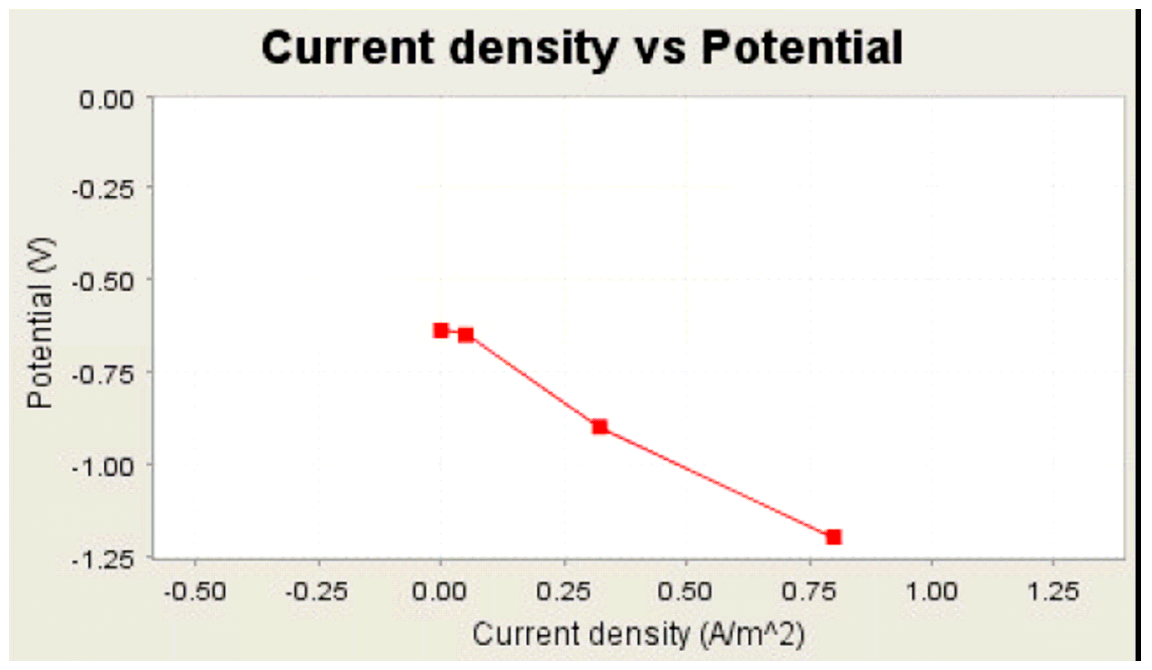

Figure 8: Polarisation curve used in the model.

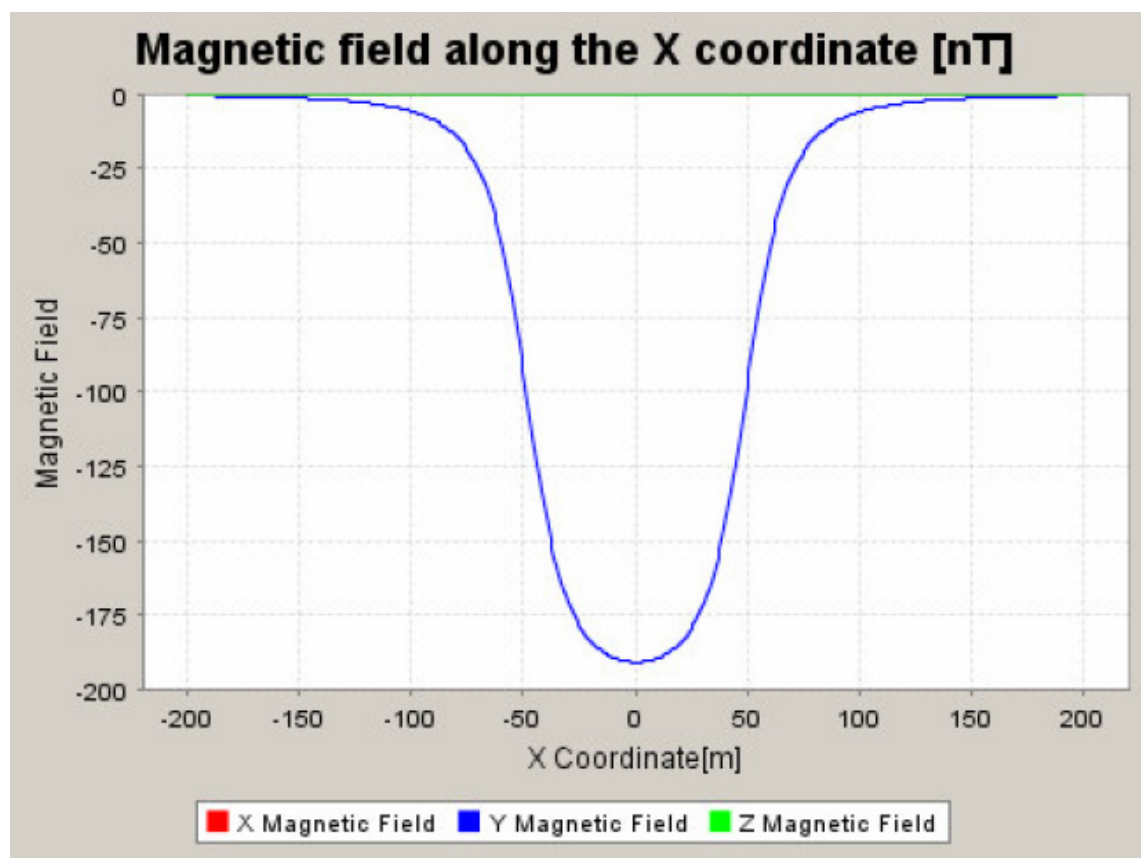

Figure 9: $\quad$ Magnetic field predicted by the structure magnetic field tool.

\section{Return Path Currents}

The magnetic field due to the currents flowing in the return path through the structure are shown in Figure 9. In this model and for the result positions chosen, the vertical and axial components are zero. 
Comparison of the transverse magnetic field along the line of sampling points is shown in Figure 10. It can be seen that the sum of the volume and structural components is very similar to the result from the dipole. Immediately underneath the middle of the hull, the dipole solution is 239.75 nanoTesla, and the sum of the results from volume and structural currents is 241 nanoTesla.

The combined magnetic field (volume currents plus return path currents) and the magnetic field from the dipole agree to within about $0.5 \%$, making the curves "Vol+struct" and "dipole" in Figure 10 indistinguishable in the figure.

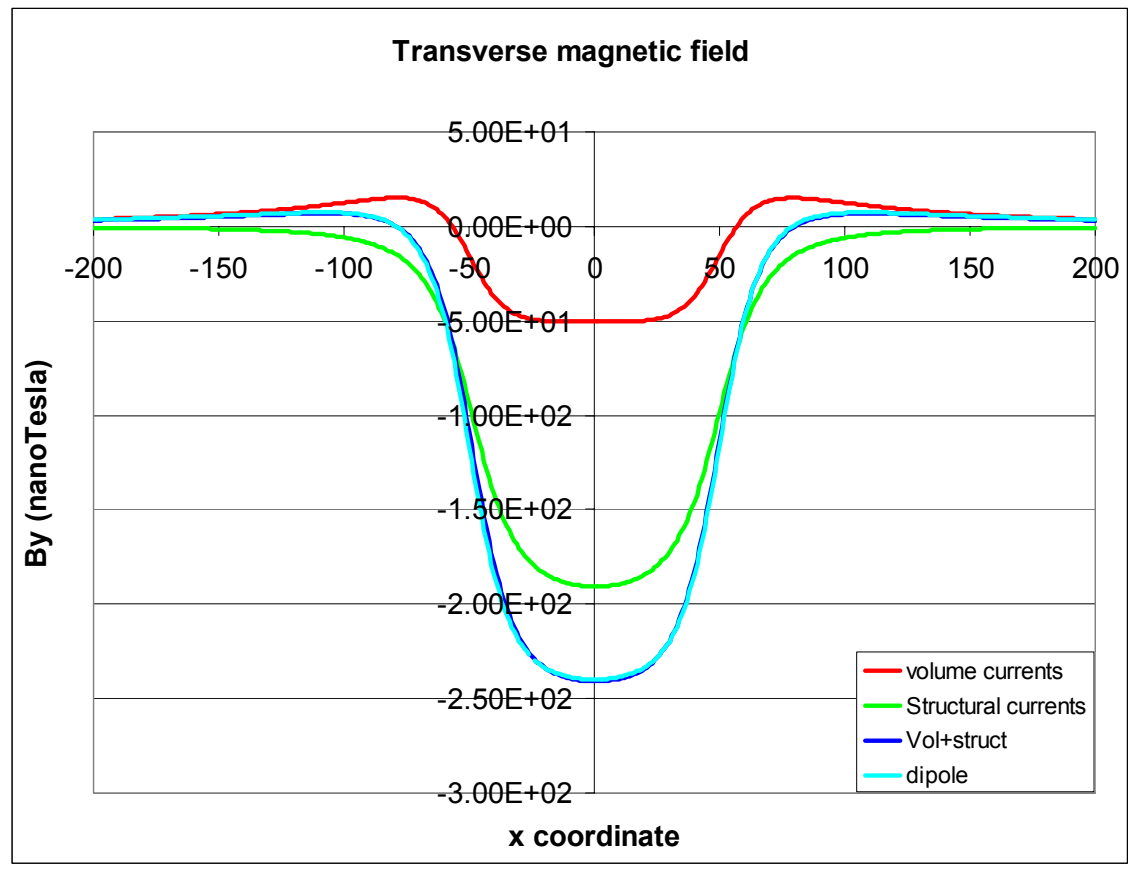

Figure 10: Comparison of magnetic field from volume currents, return path currents, and the dipole. "Vol+struct" and "dipole" curves overlap.

\section{Equivalent Dipole model}

Finally, a dipole was computed for this structure by using the Characterisation tool to match the electric signature obtained with the detailed BEM model.

The characterization tool matched the electric field as shown in Figure 11, and came up with an "equivalent dipole" with source and sink at $\mathrm{x}=+/-48$ metres, $\mathrm{y}=0$, and $\mathrm{z}=0.5$ metres.

\section{Comparison for the equivalent dipole}

The sum of the magnetic field caused by the volume currents and the magnetic field caused by the return path currents through the structure are compared with the magnetic field due to the calculated equivalent dipole in Figure 12. Immediately underneath the middle of the hull, the solution from the equivalent dipole is 238.3 nanoTesla. 


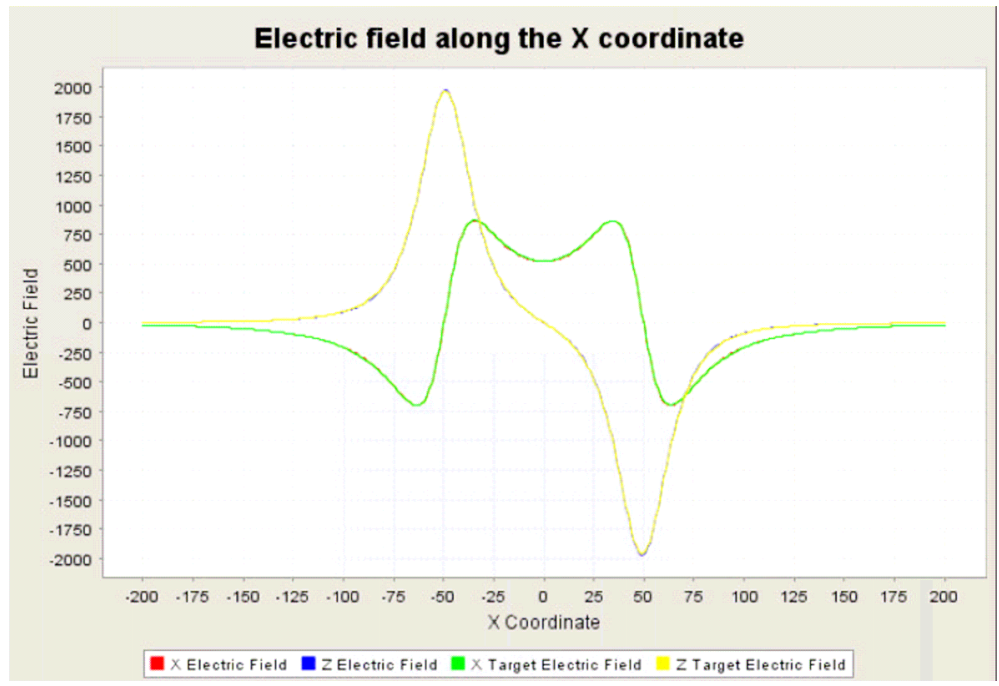

Figure 11: Comparison between the electric field computed with the BEM model and the equivalent dipole model.

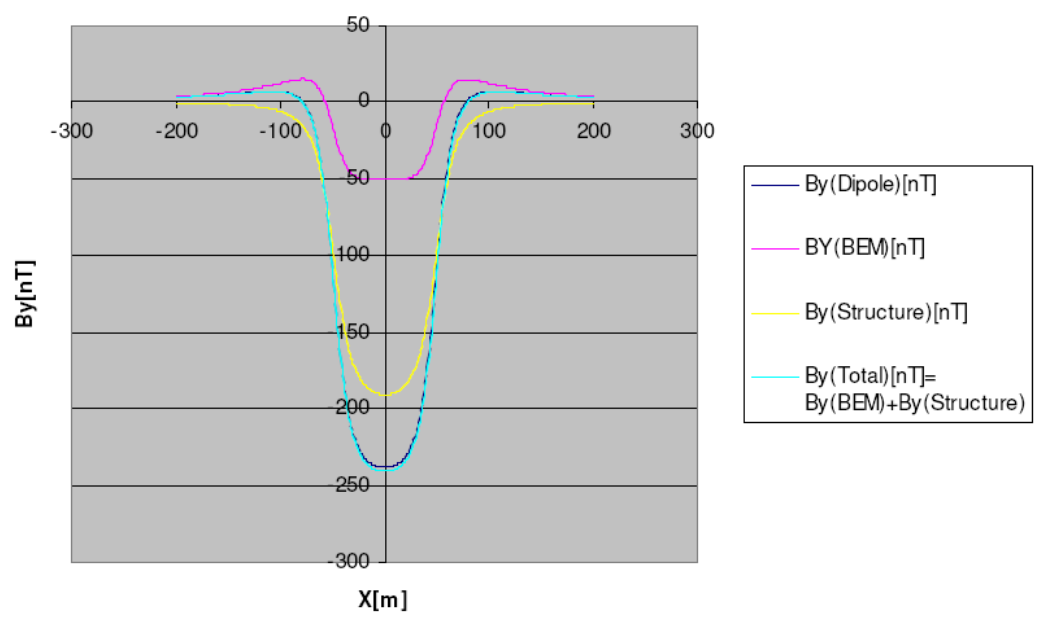

Figure 12: Comparison of magnetic field from volume currents, return path currents, and the equivalent dipole.

The magnetic field from the equivalent dipole determined by the characterization tool is within about $1 \%$ of the field from the combined structural and volume currents. 


\section{Conclusions}

It has been demonstrated that for a very simple "hull" shape calculation of magnetic fields caused by volume currents and separate calculation of magnetic fields caused by currents in the structure gives nearly the same combined result as the magnetic field calculated using a dipole. This validates the method of obtaining magnetic fields by means of detailed BEM combined with structural models.

Clearly the "detailed BEM combined with structural model" method offers most benefit when the volume current field is complicated by factors such as non-planar boundaries between the sea layer and the seabed layer(s), complex hull shape and so on.

A method has been shown of determining the equivalent dipole(s) that reproduce some target UEP (along a line of sampling points). The "target UEP" could come from a detailed BEM model of a hull, or from experimental measurements made on a range.

It has been shown that the contribution of the volume currents to the total magnetic field is significant (around $21 \%$ for the simple case studied), and so it is clear that accurate representation of the volume currents is important.

\section{References}

[1] BEASY User Guide, Computational Mechanics BEASY, Southampton, 2001.

[2] Adey R A, Niku S M, "Application of BEM in Corrosion Engineering”, Chapter in Topics in Boundary Element Research Ed, C A Brebbia, Springer Verlag, 1990.

[3] Adey R A, Baynham J M W, "Design and Optimisation of Cathodic Protection Systems Using Computer Simulation", NACE Corrosion Conference, Orlando USA, 2000.

[4] Adey R A, Pei Yuan Hang, "Computer Simulation as an aid to Corrosion Control and Reduction", NACE Corrosion Conference, San Antonio Texas USA, 1999.

[5] T. Bailey, A. Parker, R. Twelvetrees, M. Turner, S.J. Davidson. Advanced Signature Control Systems. Ultra Electronics Magnetic Division. United Kingdom. 2000.

[6] E Santana Diaz, R Adey, J Baynham, Y H Pei, Optimisation of ICCP systems to minimise electric signatures. Marelec 2001.

[7] E Santana Diaz, R Adey, B Tims. A Complete Underwater Electric and Magnetic Signature Scenario Using Computational Modeling. Marelec 2006.

[8] Allan, P.J., Ph.D. Thesis, A Study of the Electromagnetic Signatures of Marine Vessels using the Boundary Element Method, University of Glasgow, 2004. 